\title{
SEBASTIAN ZIÓŁKOWSKI
}

Université de Gdańsk

\section{Origines pré-symbolistes de l'expressionnisme dramatique : le cas de Gérard de Nerval}

\section{En guise d'introduction}

'expressionnisme dramatique fut, pendant bien longtemps, associé exclusivement à la culture germanique et l'on considérait qu'il n'exista jamais dans l'Hexagone. Un tel état de choses découlait du fait que l'esprit cartésien français était jugé foncièrement incompatible avec l'exubérance de l'art expressionniste ${ }^{1}$. Toutefois, de nos jours, cette perspective réductrice semble définitivement abandonnée grâce aux travaux de Maurice Gravier $^{2}$ et, encore davantage, ceux de Tomasz Kaczmarek. De fait, le chercheur polonais demeure le premier à avoir étudié l'œuvre d'un auteur français à l'aune de l'expressionnisme européen dans le cadre d'une thèse universitaire $^{3}$. Ainsi, à l'heure actuelle, et ceci grâce notamment à Kaczmarek, des auteurs dramatiques tels que Simon Gantillon, Henri-René Lenormand ou Jean-Victor Pellerin sont considérés comme des dramaturges profondément influencés par l'esthétique expressionniste.

${ }^{1} \mathrm{Cf}$. J.-P. Meylan, " Les expressionnistes allemands et la littérature française ", [dans :] Die Aktion. Études littéraires, 1970, vol. 3, n 3, p. 303.

${ }^{2} \mathrm{Cf}$. M. Gravier, "L'expressionnisme dramatique en France entre les deux guerres », [dans :] L'expressionnisme dans le théâtre européen, Paris, CNRS, 1971.

${ }^{3} \mathrm{Cf}$. à ce titre : T. Kaczmarek, " Henri-René Lenormand et l'expressionnisme dramatique ", [dans :] Acta Universitatis Lodziensis. Folia Litteraria Romanica, 2008, n5. 
Toutefois, nonobstant l'apparition de rares études ou articles se rapportant à l'expressionnisme dramatique en France, l'on constate toujours une pénurie presque totale de travaux qui essaieraient de repérer les origines de cet art contestataire et, dessein encore moins envisageable, qui tenteraient de les chercher en France. L'on se contente d'habitude de relever que l'art expressionniste fut profondément influencé par le symbolisme français, et les noms de Rimbaud, Lautréamont ou Maeterlinck sont parfois évoqués ${ }^{4}$. La problématique ne se trouve malheureusement pas approfondie. Pourtant, en relisant attentivement les textes des auteurs (pré-)symbolistes français, l'on remarque nombre d'affinités avec l'expressionnisme dramatique. L'un des auteurs dont l'œuvre trahit le plus de ressemblances avec l'art expressionniste est Gérard de Nerval. Aussi le présent article se veut-il être une tentative de revisiter - ne serait-ce que d'une façon brève - ses derniers écrits (Sylvie et, notamment, Aurélia) à la lumière de l'expressionnisme dramatique européen et de voir dans quelle mesure l'imaginaire nervalien semble conforme aux motifs et concepts élaborés par les auteurs expressionnistes.

\section{Le rêveur en prose}

Jean-Nicolas Illouz, I'un des plus éminents spécialistes de l'œuvre de Nerval, définit en ces termes le " noyau " de son écriture " "Il y avait là [...]" - : un "là" où l'écriture tout à la fois se penche du côté de la mort de la mère qui, en rendant la poésie impossible, a toutefois légué à la prose une insondable réserve de rêves, et invente, par contrecoup, sa voix singulière en excédant les partages formels établis et, en passant le pas, à reculons, de la modernité poétique. C'est le contretemps initial de ce "là", où

${ }^{4}$ Cf. T. Kaczmarek, Le personnage dans le drame français du XXe siècle face à la tradition de l'expressionnisme européen, Łódź, Wydawnictwo Ut, 2010, p. 59-68. 
l'écriture du "rêveur en prose" prend en charge aussi bien les déchirures de l'existence personnelle que les fractures de l'Histoire $[. ..] »^{5}$.

Il semble que toute la vie de l'auteur d'Aurélia soit hantée par ce "là ", par la douloureuse conscience d'une perte irréversible. En effet, dans l'existence de Nerval tout remonterait au traumatisme dû à la disparition fort prématurée de sa mère, événement qui influence sans doute le plus la vie de l'artiste. De sa mère, Nerval ne garde aucun souvenir : ceci est indubitablement à l'origine d'une profonde déchirure intérieure qui hantera Nerval jusqu'à sa mort suicidaire en janvier 1855 .

Dans ce gouffre d'où ressurgissent incessamment des rêves teintés d'inquiétude, voire des cauchemars du passé de même que des "souvenirs " provenant d'outre-mémoire, Nerval puise, comme dans un réservoir illimité, des images entrevues à la lisière du monde réel et de sa représentation fantasmagorique constituant pour lui la source d'inspiration créatrice. Comme le souligne Jean Giraudoux, "la communication avec un monde irréel n'est pas pour lui [Nerval] un événement rare et plein de conséquence mais un phénomène journalier ${ }^{6}$. Cependant, ses attaches avec la dimension "réelle » des choses ne sont pas totalement rompues. Cette incessante oscillation entre le plan matériel de la réalité et la recherche des significations latentes va décider de la nature d'écrits tels que Sylvie ou Aurélia. Néanmoins, il ne faut point oublier que jusqu'à un certain moment de sa carrière littéraire, Nerval semble être un homme ancré dans le réel, faisant partie des cercles littéraires de l'époque et n'ayant guère de difficulté à trouver sa juste place parmi ses confrères

5 J.-N. Illouz, Nerval " le rêveur en prose », Paris, PUF, 1997, p. 1-2.

6 J. Giraudoux, "Préface ", [dans :] G. de Nerval, Aurélia, Paris, Bibliothèque de la Pléiade, 1927, p. 16 . Les citations suivantes provenant de l'œuvre citée seront marquées à l'aide de l'abréviation $A$, la pagination après le signe abréviatif. 
littéraires ${ }^{7}$. II exerce une activité de critique dramatique, effectue des voyages, participe à des projets éditoriaux mais s'avère assez instable dans ses entreprises ${ }^{8}$. C'est à partir de 1841, l'année de la faillite spectaculaire de sa revue théâtrale Le monde dramatique, que la santé mentale de l'écrivain s'altère gravement ce qui inaugure, pour ainsi dire, le cheminement de Nerval vers un nouveau versant de son écriture.

Après sa première crise psychique, l'auteur de Sylvie s'intéresse de plus en plus aux sciences occultes, à l'ésotérisme et à la gnose. II se plonge dans la lecture de traités ésotériques, se passionne pour des religions exotiques et n'a de cesse de scruter mythes et légendes provenant de la culture populaire. Cet itinéraire particulier n'est pas sans présenter des parallèles évidents avec les pratiques spirituelles d'August Strindberg, considéré comme le précurseur de l'expressionnisme. Durant ses crises psychiques destructrices, le dramaturge suédois s'efforce lui aussi d'approfondir inlassablement ses connaissances en sciences occultes et s'inspire notamment du mysticisme théosophique et plus largement des écrits de Helena P. Blavatsky ${ }^{9}$. La pensée de l'ésotérisme théosophique selon lequel "l'intérieur possède virtuellement en germe l'extériorité, tout comme l'extériorité recèle encore les traces de l'intériorité ${ }^{10}$ est saillante dans les écrits du Suédois. Ainsi dans Un livre bleu nous pouvons lire que : " le réel

${ }^{7}$ Cf. J. Hartwig, Gérard de Nerval, Varsovie, PIW, 1972, p. 24.

8 Le parcours de Nerval en tant que journaliste et critique dramatique ainsi que son évolution dans le monde des lettres de l'époque ont été minutieusement retracés par Michel Brix. L'activité journalistique de l'auteur d'Aurélia fut très diversifiée et somme toute réussie jusqu'aux premières manifestations de la maladie mentale en 1841-42. Cf. M. Brix, Nerval journaliste. Problématique. Méthodes d'attribution, Namur, Presses Universitaires de Namur, 1989, p. 275.

${ }^{9} \mathrm{Cf}$. D. Laragné, " Du naturalisme piétiste à l'expressionnisme mystique d'August Strinberg ", [dans :] Lublin Studies in Modern Languages and Literature, 2014, vol. 38, nº 1.

10 Ibidem, p. 7. 
n'était que le reflet de ton monde intérieur et de celui des autres, c'est-à-dire que chaque homme porte vraisemblablement en lui son ciel et son enfer [...] ${ }^{11}$. Cette ressemblance semble d'autant plus patente qu'elle laisse une empreinte indélébile sur le caractère de l'œuvre de ces deux écrivains.

Pour ce qui concerne Nerval, c'est à propos d'Aurélia ou encore des Chimères ${ }^{12}$ que l'incidence de la dimension ésotérique sur sa création est le plus fréquemment évoquée. Cependant, des traces manifestes des préoccupations mystiques relevant des avatars divers des sciences occultes peuvent être repérées déjà dans Le Voyage en Orient (1951). Comme le souligne avec justesse René-Marill Albérès, "Le Voyage en Orient est déjà une œuvre ésotérique ; derrière le paysage, derrière la description plaisante et parfois badine des coutumes, s'inscrit un mystère : sous les fards et les couleurs d'un pittoresque déjà pourrissant devant l'influence de l'Occident, ces voyages méditerranéens conservent un secret : celui d'une "doctrine", d'une écriture hiéroglyphique de la vie, d'une science sacrée. Souvent Nerval ne semble être qu'un voyageur amusé qui cherche seulement le plaisir des yeux et les chatouillements de l'exotisme, et pourtant ces fraîches et prestes descriptions aboutissent toujours à cette impression bizarre d'un trésor caché, de l'Orient, trésor qui est une mystérieuse et antique recette pour lire la destinée et la vie [...]. En fin de compte, Nerval aura l'impression qu'il est allé en Orient pour devenir un in i t i é ${ }^{13}$.

${ }_{11}$ A. Strinberg, Un livre bleu, Paris, Herne, 2006, [cité d'après :] Ibidem, p. 21. 12 Chimères de Nerval demeurent une œuvre hermétique. Parmi les inspirations du poète se trouvent le tarot (La " tour abolie " dans El Desdichado étant la référence à la Tour foudroyée par Dieu, $\mathrm{XV} \mid \mathrm{e}$ arcane du tarot), la kabbale ou diverses références astrologiques. $C f$. à ce titre : A. Viatte, "Mysticisme et poésie chez Gérard de Nerval », [dans :] Cahiers de l'Association internationale des études françaises, 1963, n 15.

13 R.-M. Albérès, Gérard de Nerval, Paris-Bruxelles, Éditions Universitaires, 1955, p. 43-44. 
Ainsi sommes-nous en présence d'un procédé pouvant être considéré comme une véritable charpente de la poétique nervalienne qui consiste à percevoir derrière la déroutante apparence matérielle des choses la "vraie " nature des phénomènes. En d'autres termes, "il [Nerval] entrevoit la possibilité d'une double interprétation des choses ${ }^{14}$. II s'agit là d'une attitude - tant existentielle que créatrice - qui nous renvoie logiquement aux desseins artistiques des symbolistes, mais qui nous fait penser encore davantage à la production des auteurs expressionnistes. Ces derniers, à l'instar de Nerval - ancré profondément dans l'esthétique romantique et annonçant déjà, notamment avec Aurélia et Chimères, l'avènement du symbolisme -, délaissent la réalité palpable pour se lancer dans la découverte d'un monde caché. Ils tournent le dos à la mimèsis aristotélicienne pour sonder les précipices d'un monde fragmenté par le prisme de la conscience égarée de l'artiste.

Ce qui rapprocherait Nerval des expressionnistes, c'est l'exploitation du motif du rêve, la technique principale à l'appui de laquelle ils envisagent la désobjectivisation progressive de l'univers qu'ils présentent à leur lecteur. À cet égard, il ne nous reste qu'à mentionner que tant $A u$ rélia de Nerval que les écrits de Strindberg (Inferno, La Sonate des spectres, et, en tout premier lieu, Le Songe, un jeu de rêves) préfigurent déjà les acquis de la psychanalyse de Freud, constituant une source d'inspiration inépuisable pour les expressionnistes tout comme pour les surréalistes. Déjà au commencement de cette rêverie visionnaire qu'est Aurélia, le lecteur se trouve proprement immergé dans la description d'un état plein d'hésitation, se situant à mi-chemin entre le sommeil et la veille et qui semble être une anticipation encore à l'état brut, dirait-on, mais assurément empreinte de génie des théories freudiennes. Ainsi, Nerval décrit déjà à sa manière le travail de l'esprit

14 Ibidem, p. 41. 
qui se soustrait au contrôle de la raison dans l'état de veille et d'assoupissement :

Les premiers instants du sommeil sont l'image de la mort ; un engourdissement nébuleux saisit notre pensée, et nous ne pouvons déterminer l'instant précis où le moi, sous une autre forme, continue l'œuvre de l'existence. C'est un souterrain vague qui s'éclaire peu à peu, et où se dégagent de l'ombre et de la nuit les pâles figures gravement immobiles qui habitent le séjour des limbes. Puis le tableau se forme, une clarté nouvelle illumine et fait jouer ces apparitions bizarres; - le monde des Esprits s'ouvre pour nous. $(A, 25-26)$

II ne faut point oublier que les expressionnistes, plus encore que les surréalistes, étaient toujours, et d'une manière toute particulière, admiratifs des avancées de la psychanalyse. Lorsque l'on prend en considération les idées articulées par l'auteur d'Aurélia, l'on se rend compte que ses correspondances avec l'œuvre de Strindberg et, encore peut-être plus perceptiblement, avec celle d'HenriRené Lenormand sont frappantes. Nous pensons ici en particulier à L'homme et ses fantômes (1925) ${ }^{15}$, histoire d'un séducteur aux penchants homosexuels latents, un être à vaste complexité identitaire entouré de personnages-satellites étant la projection de ses forces psychiques, de ses divers "moi " qui se doublent et se dédoublent. C'est, semble-t-il, dans l'œuvre de Lenormand, la pièce trahissant le plus de convergences avec la pensée du psychiatre viennois. Armand Salacrou, avec sa pièce maîtresse L'Inconnue d'Arras (1935) ${ }^{16}$, pourrait, lui aussi, se retrouver dans le même sillage. Dans L'Inconnue, Salacrou met en scène le délire mortuaire d'un suicidé et instaure un univers baignant dans une ambiance onirique : les événements narrés semblent flotter dans un monde où le temps est totalement atomisé et dans lequel l'espace n'est plus celui « d'un environnement, mais la projection

${ }^{15}$ Cf. H.-R. Lenormand, L'homme et ses fantômes, [dans :] Idem, L'Homme et ses fantômes, À l'Ombre du mal, Théâtre complet 4, Paris, G. Crès et Cie, 1925.

${ }^{16}$ Cf. A. Salacrou, L'Inconnue d'Arras, Paris, Gallimard, 1968. 
du Moi du personnage ${ }^{17}$. Ces affinités prouvent en quelque sorte la perpétuation d'une nouvelle conception de l'art annoncée par Nerval.

L'art nervalien s'inscrirait bien dans ce que Maurice Gravier qualifie de " féerie expressionniste ". D’après cet éminent connaisseur de l'expressionnisme européen, " elle [la féerie expressionniste] débouche sur l'ambiguïté, l'ambivalence [...], l'angoisse, elle fait de nous des étrangers dans le monde ${ }^{18}$. Ladite féerie devient possible, tout comme dans les drames expressionnistes, grâce à une rupture décidée avec l'entendement traditionnel des notions de temps et d'espace. Ceci est d'autant plus important du point de vue de nos considérations que " nul autre art n'a consacré autant de programmes au temps et à l'espace que l'expressionnisme ${ }^{19}$. La féerie expressionniste, pareillement à la féerie nervalienne ${ }^{20}$, en faisant $d u$ rêve son fer de lance dans la lutte contre la mimèsis dépassée et les conventions d'antan, permet d'effectuer de véritables voyages, des "sauts" dans le temps et l'espace. Dans Aurélia, Nerval accède à une autre dimension de l'existence qui ne peut être atteinte qu'au moyen d'une " extension » temporelle et spatiale :

Je n’ai jamais éprouvé - avoue Nerval - que le sommeil fût un repos. Après un engourdissement de quelques minutes, une vie nouvelle commence, affranchie des conditions du temps et de l'espace, et pareille

17 T. Kaczmarek, « Entre le temps de Kant et le temps d'Einstein, ou requête pour un texte expressionniste français " [dans :] Etudes romanes de Brno, 2010, n³1, p. 48.

$18 \mathrm{M}$. Gravier, L'expressionnisme dramatique en France entre les deux guerres, [cité d'après :] T. Kaczmarek, "Simon Gantillon et l'expressionnisme dramatique ", [dans :] Etudes romanes de Brno, 2016, n 37, p. 128.

${ }^{19}$ T. Kaczmarek, "Entre le temps de Kant et le temps d'Einstein », op. cit., p. 58.

20 Le monde aux allures féeriques se laisse le mieux sentir dans Sylvie, récit dans lequel le merveilleux et le légendaire s'entremêlent : le monde présenté s'étend à mi-chemin entre le rêve et le réel, la dimension onirique participant de la désobjectivisation de l'univers esquissé par Nerval. Cf. à cet égard : P. Laforgue, "Féeries nervaliennes ", [dans :] Féeries, 2015, n 12, p. 40. 
sans doute à celle qui nous attend après la mort. Qui sait si n'existe pas un lien entre ces deux existences et s'il n'est pas possible à l'âme de les nouer dès à présent ? $(A, 164)$

Pour Nerval, comme pour les expressionnistes, le rêve permet de se libérer, bien que temporairement, des contraintes de la matérialité et de l'entendement ordinaire du temps. Comme les expressionnistes, Nerval semble déployer la possibilité d'une relativité de plus en plus poussée de différentes temporalités parallèles qui fait affront à toute conceptualisation du temps s'appuyant sur la chronologie et la linéarité. Les expressionnistes, eux aussi, en se servant - entre autres procédés - de la technique du rêve, " s'affranchissent de vieilles notions spatiales tout en proposant le règne de l'espace intérieur. Ils s'éloignent aussi de la réalité pour scruter en profondeur l'intériorité de la vie ${ }^{21}$.

Nerval, comme les expressionnistes, exploite le motif du rêve pour instaurer une réalité potentielle ou alternative dans laquelle pourrait se déployer une temporalité toute relative présentant, selon nous, quelques affinités avec le temps einsteinien qui, tout en se libérant des notions chronologiques et spatiales périmées, serait le plus propice à définir une réalité parallèle, celle du rêve. Un tel procédé se retrouve entre autres dans l'œuvre de Strinberg dans laquelle, aux dires de Kaczmarek, le temps " correspond à la matrice d'un des mondes potentiels régi et gouverné par ses propres lois, le cas échéant, par les visions de celui qui rêve ${ }^{22}$. Même si les surréalistes, eux

${ }^{21} \mathrm{~T}$. Kaczmarek, "Entre le temps de Kant et le temps d'Einstein ", op. cit., p. 48. D'ailleurs, pour s'en convaincre, l'on n'a qu'à découvrir Le temps est un songe de Lenormand, drame dans lequel à travers la déréalisation de l'univers présenté, l'entendement traditionnel de la temporalité est abandonné au profit d'un continuum temporel dans lequel passé, présent et futur ne sont plus que des notions purement intellectuelles : "Mourir c'est [...] atteindre [...] cette marche où tout coexiste ". Cf. H.-R. Lenormand, Le temps est un songe, [dans :] Idem, Les Ratés, Le Temps est un songe, Théâtre complet 1, Paris, Albin Michel, 1921, p. 213.

22 T. Kaczmarek, "Entre le temps de Kant et le temps d'Einstein », op. cit., p. 50. 
aussi, se servent à volonté de ce motif, ils n'ambitionnent souvent que $d^{\prime}$ " épater le public ${ }^{23}$, de produire un choc et garantir ainsi le sentiment du renouveau tout en profitant de l'ahurissement causé par la sensation de l'originalité : I'exploitation du motif du rêve n'entraîne pas dans leurs drames une profonde discontinuité et déstructuration spatio-temporelles. La profondeur " nervalienne " s'en trouve donc à proprement parler absente. Ce sont les œuvres expressionnistes qui l'exploitent en profondeur, allant parfois jusqu'à mettre en doute l'existence même du temps (l'entendement kantien du temps) et de l'espace tout en optant pour le caractère illusoire du monde. Dans Le Temps est un songe de Lenormand, Nico tient ces propos définissant l'essence des desseins expressionnistes et qui semblent correspondre à l'écriture de Nerval : " Hier, aujourd'hui, demain, ce sont des mots [...]. Des mots qui n'ont de réalité que pour nos mesquines cervelles. Hors d'elles, il n'y a ni passé, ni avenir... Rien qu'un immense présent ${ }^{24}$.

\section{La figure du double}

Le mythe du double marqua profondément l'œuvre de Nerval. Son exploitation récurrente témoigne, d'une part, d'une imprégnation profonde de l'écriture nervalienne de vieux mythes provenant de la culture allemande populaire, de l'autre, elle trouve son explication dans la personnalité vague et fluctuante d'un malheureux égaré et harcelé par les épreuves de la maladie mentale. La réalisation de ce motif est, dans l'écriture nervalienne, pluridimensionnelle. Comme le remarque à très juste titre Jacinthe Rouisse, chez Nerval le motif du double peut être considéré à des niveaux différents de l'analyse " selon qu'il est lié au héros (il), aux héroïnes (elles) ou au narra-

23 Ibidem, p. 49.

${ }^{24} \mathrm{H}$.-R. Lenormand, Le temps est un songe, op. cit., p. 213. 
teur-héros (moi) ${ }^{25}$. Ainsi le double d'un héros considéré à la troisième personne pourrait-il être repéré dans Le Voyage en Orient (notamment dans L'histoire du calife Ha$k e m)^{26}$, le sosie des héroïnes dans Sylvie, et enfin, celui de Nerval lui-même dans Aurélia ${ }^{27}$. Et c'est justement ce dernier cas qu'il nous convient de soumettre à une analyse approfondie car sa ressemblance avec le concept de double élaboré par les auteurs expressionnistes est sans conteste la plus frappante.

Ainsi, Nerval recourt à un dédoublement du héros de son récit, récit dans lequel la distinction entre l'auteur et le narrateur n'est guère aisée. Le narrateur-héros nervalien, constitue, tout à fait comme dans les œuvres dramatiques expressionnistes, la projection du moi de l'auteur sur l'instance textuelle, correspondant au principe expressionniste d'irradiation du $\mathrm{moi}^{28}$ selon lequel "le moi de l'auteur-démiurge irradie dans toute son œuvre, en s'infiltrant dans les moindres détails de son drame ${ }^{29}$. Le lecteur peut donc, tout en dérogeant à de nombreux préceptes relevant de la théorie littéraire, recourir à un rapprochement de ces deux instances, deux catégories divergentes et se situant à un niveau différent de l'analyse littéraire (catégories extratextuelle et intratextuelle). Toutefois, dans le cas de l'auteur d'Aurélia, tout comme dans celui des expressionnistes, ce dédoublement peut être compris de deux manières. D'un côté, comme nous venons de le signaler, il s'agit, toutes proportions gardées,

25 J. Rouisse, La figure du double chez Gérard de Nerval, Montréal, McGill University, 1983, p. 3.

${ }^{26}$ Ibidem, p. 23-25.

27 J. Hartwig, Gérard de Nerval, op. cit., p. 44-47. Cf. également J. Richer, "Nerval devant la psychanalyse ", [dans :] Cahiers de l'Association internationale des études françaises, 1955, no 7, p. 63.

${ }^{28}$ Cf. T. Kaczmarek, "Entre le temps de Kant et le temps d'Einstein ", op. cit., p. 49. Cf. également G. Szewczyk, Strindberg jako prekursor ekspresjonizmu w dramacie, Katowice, Wydawnictwo UŚ, 1984, p. 9.

${ }^{29} \mathrm{~T}$. Kaczmarek, Le personnage dans le drame français du XXe siècle face à la tradition de l'expressionnisme européen, op. cit., p. 103. 
du double de l'auteur, de l'autre, le double de l'auteur va, lui aussi, se heurter à son sosie. Nous avons donc affaire au " dédoublement du double ", procédé fort caractéristique de l'expressionnisme dramatique ${ }^{30}$. Voici comment le narrateur-héros semble entrevoir pour la première fois son double et dans quel état cet événement le plonge:

Couché sur un lit de camp, j'entendais que les soldats s'entretenaient d'un inconnu arrêté comme moi et dont la voix avait retenti dans la même salle. Par un singulier effet de vibration, il me semblait que cette voix résonnait dans ma poitrine et que mon âme se dédoublait pour ainsi dire, - distinctement partagée entre la vision et la réalité. Un instant j'eus l'idée de me retourner avec effort vers celui dont il était question, puis je frémis en me rappelant une tradition bien connue en Allemagne, qui dit que chaque homme a un double, et que lorsqu'il le voit, la mort est proche. $(A, 38)$

Comme nous le voyons, l'apparition du double provoque chez le narrateur une réaction d'inertie et de recul, il ne se trouve point la force de jeter sur lui le plus insignifiant des regards. Un tel comportement peut être représentatif de l'angoisse d'être confronté à sa propre image, d'entrevoir, comme dans un miroir, le reflet de son visage, de son moi appréhendé. Le refus de faire face à sa propre image nous en dit long sur sa crise identitaire profonde. L'apparition du double du narrateur découle d'une subjectivisation illimitée motivée et, dirait-on, justifiée, par le caractère manifestement visionnaire du récit. Nous serions par conséquent en présence d'un motif caractéristique de l'esthétique expressionniste car la réalité dans le cas du récit nervalien n'est plus perçue qu'en fonction des fluctuations du psychisme égaré du narrateur-héros. Cependant, selon Rouisse, cette subjectivisation se plierait également à un autre dessein. Ainsi, dans son optique, " le modèle du double participe [...] dans Aurélia de l'expérience la plus ambitieuse du narrateur-héros, celle qui consiste à transformer son existence en mythe, à forcer

${ }^{30}$ Cf. Ibidem, p. 128-131. 
"ces portes mystiques" de la "Jérusalem céleste" dans laquelle, du narrateur "fou" qu'il était au début du texte, il se métamorphose à la fin en messie annonçant "I'heureuse nouvelle" ${ }^{31}$.

De même que dans les drames expressionnistes mais aussi dans ceux de Strindberg, la rupture délibérée avec toute peinture réaliste et fidèle de la réalité joue un rôle important. De fait, c'est justement à travers l'intériorisation de la perception du monde, à travers toutes les épreuves que provoque la conscience de sa nature double que le narrateur-héros semble parvenir à retrouver son unité illusoire. II s'acharne à trouver la signification profonde de ses errements, à les doter d'une cohérence, les justifier d'une certaine manière. Le récit nervalien acquiert ainsi le caractère d'une aventure initiatique. À l'instar des textes ésotériques et traités occultes anciens, pour atteindre la vérité, il faut s'affranchir définitivement du carcan de la logique régissant les masses, il faut opter pour ce qui se trouve habituellement qualifié de déraison car c'est justement la rationalité dépassée qui prive l'homme de la connaissance de l'essence des choses.

Pour ce qui est de l'itinéraire initiatique que suit le narrateur-héros, il s'agit toujours, comme cela a déjà été signalé plus haut, d'une quête identitaire, thème d'une importance cruciale dans l'écriture de l'auteur d'Aurélia. Ainsi, à travers la dissolution presque complète de la personnalité que favorise la plongée dans un monde du rêve rendu multidimensionnel grâce au syncrétisme religieux et culturel, le héros-narrateur nervalien se sent enfin en mesure de découvrir son vrai moi, de le retrouver dans sa pleine multiplicité. Remarquons qu'en scrutant son for intérieur pour retrouver des repères identitaires, Nerval recourt à la théorie de la migration des âmes, motif particulièrement chéri des expressionnistes. Dans Bifur de Simon Gantillon, pour nous servir de cet exemple, l'esprit

31 J. Rouisse, La figure du double chez Gérard de Nerval, op. cit., p. 97. 
de la Reine renaît dans le corps de la jeune Claire, s'amalgamant dans le destin de la malade au seuil de l'agonie, l'aspect salvateur de cette intervention surnaturelle étant ainsi mis en relief ${ }^{32}$. Le narrateur-héros nervalien semble également quêter sa délivrance symbolique lorsqu'à un moment donné il sent vivre en lui l'âme de Napoléon. Bien qu'il ne se croie aucunement être le grand Empereur, il a besoin de raviver en lui les souvenirs de ses incarnations antérieures en vue de s'approprier en quelque sorte leur éclat et magnificence. Un tel procédé participe de la dimension de son écriture qui est aujourd'hui - semble-t-il - la plus étudiée par la critique nervalienne, à savoir d'un art d'écrire considéré comme une thérapie ou un échappatoire aux troubles de la vie psychique. Les cercles d'études nervaliennes s'efforcent donc d'élucider son parcours littéraire à l'aune des approches "psycho-psychanalytiques "33. Ainsi, à la lumière des considérations évoquées ci-dessus, nous voyons que l'exploitation téméraire du motif du double mène le narrateur-héros, d'une part, au ressassement de sa complexité identitaire, de l'autre, elle constitue la pénétration d'un monde spirituel nourri de traditions occultes, mais aussi, comme chez Strindberg, de préceptes bouddhistes. Le suédois, lui aussi, est admiratif des idées bouddhistes d'après lesquelles " les doubles existent et [...] sont bien

\footnotetext{
${ }^{32}$ Cf. S. Gantillon, Bifur, Paris, Librairie théâtrale, 1932, p. 59-62. L'exploitation de ce motif est aussi particulièrement accomplie dans L'Homme et ses fantômes d'Henri-René Lenormand, drame où le spiritisme joue un rôle majeur dans le cadre des ressassements identitaires de L'Homme. Cf. H.-R. Lenormand, L'Homme et ses fantômes, op. cit.

${ }^{33}$ Cf. H. Mizuno, "Corps et âme dans "Aurélia" de Gérard de Nerval ", [dans :] Romantisme, 2005, no 127, p. 59-77. Cf. également J. Richer, "Nerval devant la psychanalyse ", [dans :] Cahiers de l'Association internationale des études françaises, 1955, $\mathrm{n}^{\circ}$ 7. Pour en savoir plus sur la dimension thérapeutique qu'avait pour Nerval l'écriture $c f$. J.-L., Steinmetz, "Les rêves dans "Aurélia" de Gérard de Nerval ", [dans :] Littérature, 2010, nº 158.
} 
cachés pour l'esprit rationaliste ${ }^{34}$. Pour les découvrir il est donc impératif de s'écarter le plus possible de la rigueur rationaliste du cogito cartésien.

\section{La primauté de l'âme}

Le protagoniste que nous fait découvrir Nerval, notamment dans ses derniers écrits datant des années 50 , commence à ressentir douloureusement l'estompement des traits de la réalité. Déjà dans Sylvie il est hanté par les souvenirs, il part à la recherche des joies enfantines et entend résonner distinctement "un écho lointain des fêtes naïves de la province ${ }^{35}$. Pourtant si dans Sylvie Nerval s'aventure dans la découverte des instants passés d'une vie qui n'est plus, ce n'est que dans Aurélia que la quête à laquelle s'adonne le narrateur-héros prend l'ampleur d'une véritable aventure d'initiation. Dans Sylvie, cependant, l'on pourrait déjà déceler une dimension spirituelle et onirique manifestes d'une œuvre qui erre, hésite, essaie de trouver sa voie. Selon Jean-Luc Steinmetz, c'est dans Sylvie que le rêve " commence déjà à avoir droit de cité ${ }^{36}$.

Les jalons d'une écriture subjectiviste et onirique se trouvent donc posés. Désabusé et perdu, le narrateur-héros entreprend dans son testament littéraire qu'est Aurélia son inlassable marche à l'étoile ${ }^{37}$ initiant symboliquement l'apogée de sa crise psychique. II s'efforce de retrouver son identité mais est en même temps conscient du caractère vain de cette entreprise. Comment pourrait-il en effet parvenir à un quelconque repérage identitaire viable lorsque

34 T. Kaczmarek, Le personnage dans le drame français du XXe siècle face à la tradition de l'expressionnisme européen, op. cit., p. 108.

${ }^{35}$ G. de Nerval, Sylvie, Paris, À la librairie illustrée, 1892, p. 6.

36 J.-L. Steinmetz, "Les rêves dans "Aurélia" de Gérard de Nerval ", op. cit., p. 105.

${ }^{37}$ Allusion également au brillant ouvrage de Corinne Bayle. Cf. C. Bayle, Gérard de Nerval. La marche à l'étoile, Ceyzérieu (Ain), Champ Vallon, 2001. 
toute la réalité se trouve déjà déformée par le prisme de son psychisme embrouillé ? II ne lui reste plus à vrai dire qu'à donner libre cours au déploiement de l'âme restituant à l'individu les domaines de la spiritualité et de l'inconscient, l'âme étant ainsi l'unique bouclier qui lui reste contre le tragique de son destin. L'âme possède un rôle cardinal à jouer car elle symbolise le désir qu'éprouve Nerval de se libérer des attaches de l'enveloppe corporelle en vue de retrouver la nature divine de l'homme. Voici le ressassement perpétuel de ce désir de délivrance que l'on retrouve notamment dans Aurélia:

Etendu sur un lit de camp, je crus voir le ciel se dévoiler et s'ouvrir en mille aspects de magnificences inouïes. Le destin de l'âme délivrée semblait se révéler à moi comme pour me donner le regret d'avoir voulu reprendre pied de toutes les forces de mon esprit sur la terre que j'allais quitter... D'immenses cercles se traçaient dans l'infini, comme les orbes que forme l'eau troublée par la chute d'un corps ; chaque région, peuplée de figures radieuses, se colorait, se mouvait et se fondait tour à tour, et une divinité, toujours la même, rejetait en souriant les masques furtifs de ses diverses incarnations [...]. $(A, 132)$

Chez Nerval, la plongée dans l'univers du divin s'inscrirait, comme dans le cas des dramaturges expressionnistes, dans le contexte du romantisme allemand. Pour ce qui est de Nerval, ce désir romantique de la délivrance de l'âme se trouve nourri d'une profondeur du regard hors du commun, ce qui d'autre part s'explique par le fait que l'auteur d'Aurélia traduisit les auteurs allemands et les fréquenta. L'emprise qu'ont sur lui les romantiques allemands (Goethe, Schiller, Novalis) fait que Nerval, en admirateur fidèle, emprunte également " son cadre [celui de l'art allemand] conceptuel : "romantisation", [...] "inachèvement", "fragmentation" et "infinitisation" de l'œuvre ${ }^{38}$. La prose et la poésie nervaliennes, à l'instar de celles d'un Novalis, suivent la voie qui mène vers l'intérieur. Le poète allemand ne disait-il pas que "le chemin mystérieux va

38 A. Moioli, " "Romantiser" la vie : Aurélia de Nerval ", [dans :] Acta fabula, 2013, vol. 16, n 1 : http://www.fabula.org/acta/document9095.php. 
vers l'intérieur [et que] c'est en nous, sinon nulle part, qu'est l'éternité avec ses mondes, le passé et l'avenir " $^{39}$ ? Comme Nerval, l'auteur des Hymnes à la nuit n'hésite pas à sonder les recoins incandescents de l'âme humaine et procède à une intériorisation qui seule peut l'éloigner - ne serait-ce que de façon hautement imparfaite - de la menace que constitue la finitude à laquelle se trouve soumis le corps.

En adoptant une pareille optique, Nerval érige le corps humain en symbole de la déchéance et décrépitude ${ }^{40}$ subordonnées, entre autres choses, aux lois immuables du temps et de l'espace. Une pareille vision du corps ne serait nullement étrangère aux expressionnistes. Déjà dans les drames strindbergiens, l'épanchement de l'âme servait à une désobjectivisation poussée de l'univers présenté. Et Tomasz Kaczmarek de constater que la recherche de l'âme à laquelle s'adonnent les expressionnistes s'explique par "le désir de se débarrasser du joug du corps ${ }^{41}$. Par ailleurs, l'exégèse de l'âme expressionniste qu'esquisse Kaczmarek dans son étude nous semble avoir été dédiée à Nerval. Ainsi, aux dires du chercheur, "l'âme expressionniste se prête à diverses interprétations. Tout d'abord elle est le mot d'ordre d'une génération qui, désabusée des imperfections du monde, se replie sur la spiritualité de l'homme. L'âme comprise de cette manière signifie la confiance en la régénération de l'humanité qui, aux yeux des artistes de l'époque, avait rompu ses attaches avec le mysticisme [...]. Mais l'âme, une fois dé-

39 Propos de Novalis (dans la traduction d'Albert Béguin) cités d'après : A. Béguin, L'Âme romantique et le rêve, Marseille, Actes Sud, 1937, p. 273.

40 Le corps, dans Aurélia, est associé entre autres au sentiment d'impureté : "Vers deux heures on me mit au bain, et je me crus servi par les Walkyries, filles d'Odin, qui voulaient m'élever à l'immortalité en dépouillant peu à peu mon corps de ce qu'il avait d'impur » (G. de Nerval, Aurélia, op. cit., p. 124).

${ }^{41} \mathrm{~T}$. Kaczmarek, Le personnage dans le drame français du XXe siècle face à la tradition de l'expressionnisme européen, op. cit., p. 122. 
couverte, peut également être anxiogène car l'homme se rend compte de son statut éphémère et insaisissable ${ }^{42}$.

Pareillement donc aux perspectives instaurées par Nerval, dans les œuvres des expressionnistes (notamment dans les drames de Hjalmar Bergman ou ceux de Pär Lagerkvist) ${ }^{43}$ il est fort souvent question d'un certain risque que peut entraîner la plongée téméraire dans les profondeurs de l'âme. D'une part, elle constitue un échappatoire aux contraintes corporelles, mais, de l'autre, elle peut se muer en menace de déraison. Cependant, une pareille tentative constitue pour le narrateur-héros nervalien, tout comme pour le personnage des drames expressionnistes, l'unique issue pour se libérer des troubles identitaires constants.

Quoi qu'il en soit, l'âme occupe dans le dernier récit de Nerval - même si elle n'est pas toujours désignée explicitement - une place prépondérante. Pourtant, combien tragique semble cette inlassable recherche de la spiritualité à travers la libération de l'âme du joug de la corporalité lorsque l'on connaît le dénouement de l'existence de l'auteur d'Aurélia. Déjà quelques mois après la disparition tragique de notre auteur, Baudelaire n'évoquait-il pas " un écrivain d'une honnêteté admirable, d'une haute intelligence [...] qui fut toujours lucide [...] et qui est allé discrètement, sans déranger personne - et si discrètement que sa discrétion ressemblait à du mépris - délier son âme dans la rue la plus noire qu'il pût trouver $»^{44}$ ? Nerval aurait de cette manière libéré son âme tout en accomplissant le rêve exprimé par l'attitude du narrateur-héros dans Aurélia.

\footnotetext{
42 Ibidem, p. 123.

${ }^{43} \mathrm{Cf}$. Ibidem.

${ }^{44} \mathrm{Ch}$. Baudelaire, Edgar Poe, sa vie et ses œuvres, [cité d’après :] J.-P. Bertrand, P. Durand, La modernité romantique : de Lamartine à Nerval, ParisBruxelles, Les impressions nouvelles, 2006, p. 195.
} 


\section{Conclusion}

À la lumière des considérations ci-dessus, force est de constater que les desseins artistiques de Nerval, les motifs qu'il exploite de même que ses inspirations demeurent étonnamment convergents avec ceux qui furent propres à Strindberg et, par la suite, aux dramaturges créant dans le sillage de l'expressionnisme européen. Ces affinités saillantes font de Nerval sinon l'un des précurseurs lointains de l'expressionnisme dramatique, du moins un auteur auquel les expressionnistes pourraient vouer respect. Cette perspective inédite serait à même d'inciter les chercheurs à s'astreindre au joug d'études approfondies pouvant mener à des conclusions surprenantes. 


\section{bibliographie}

Albérès R.-M., Gérard de Nerval, Paris-Bruxelles, Éditions Universitaires, 1955.

Bayle C., Gérard de Nerval. La marche à l'étoile, Ceyzérieu (Ain), Champ Vallon, 2001.

Béguin A., L'Âme romantique et le rêve, Marseille, Actes Sud, 1937.

Bertrand J.-P., Durand P., La modernité romantique : de Lamartine à Nerval, Paris-Bruxelles, Les impressions nouvelles, 2006.

Brix M., Nerval journaliste. Problématique. Méthodes d'attribution, Namur, Presses Universitaires de Namur, 1989.

Gantillon S., Bifur, Paris, Librairie théâtrale, 1932.

Gravier M., "L'expressionnisme dramatique en France entre les deux guerres ", [dans :] L'expressionnisme dans le théâtre européen, Paris, CNRS, 1971.

Hartwig J., Gérard de Nerval, Varsovie, PIW, 1972.

Illouz J.-N., Nerval, "le rêveur en prose ", Paris, PUF, 1997.

Kaczmarek T., « Entre le temps de Kant et le temps d'Einstein, ou requête pour un texte expressionniste français ", [dans :] Études romanes de Brno, 2010, no 31.

Kaczmarek T., " Henri-René Lenormand et l'expressionnisme dramatique ", [dans :] Acta Universitatis Lodziensis. Folia Litteraria Romanica, 2008, n 5.

Kaczmarek T., Le personnage dans le drame français face à la tradition de l'expressionnisme européen, Łódź, Wydawnictwo Ut, 2010.

Kaczmarek T., "Simon Gantillon et l'expressionnisme dramatique ", [dans :] Etudes romanes de Brno, 2016, nº 37.

Laforgue P., "Féeries nervaliennes ", [dans :] Féeries, 2015, n 12.

Laragné D., " Du naturalisme piétiste à l'expressionnisme mystique d'August Strinberg ", [dans :] Lublin Studies in Modern Languages and Literature, 2014, vol. 38, n० 1.

Lenormand H.-R., Le temps est un songe, [dans :] Idem, Les Ratés, Le Temps est un songe, Théâtre complet 1, Paris, Albin Michel, 1921.

Lenormand H.-R., L'Homme et ses fantômes, [dans :] Idem, L'Homme et ses fantômes, A l'Ombre du mal. Théâtre complet 4, Paris, G. Crès et Cie, 1925. Meylan J.-P., "Les expressionnistes allemands et la littérature française ", [dans :] Die Aktion. Études littéraires, 1970, vol. 3, nº 3.

Mizuno H., "Corps et âme dans "Aurélia" de Gérard de Nerval », [dans :] Romantisme, 2005, n 127.

Moioli A., " "Romantiser" la vie : Aurélia de Nerval », [dans :] Acta fabula, 2013, vol. 16, no 1 : http://www.fabula.org/acta/document9095.php.

Nerval G. de, Aurélia, [avec introduction de Jean Giraudoux], Paris, Bibliothèque de la Pléiade, 1927.

Nerval G. de, Sylvie, Paris, À la librairie illustrée, 1892.

Richer J., "Nerval devant la psychanalyse ", [dans :] Cahiers de l'Association internationale des études françaises, 1955, n० 7.

Rouisse J., La figure du double chez Gérard de Nerval, Montréal, McGill University, 1983.

Steinmetz J.-L., "Les rêves dans "Aurélia" de Gérard de Nerval », [dans : ] Littérature, 2010, nº 158. 
Strindberg A., Un livre bleu, Paris, Herne, 2006.

Szewczyk G., Strindberg jako prekursor ekspresjonizmu w dramacie, Katowice, Wydawnictwo UŚ, 1984.

Viatte A., " Mysticisme et poésie chez Gérard de Nerval », [dans :] Cahiers de l'Association internationale des études françaises, 1963, no 15.

\section{abstract}

\section{Pre-symbolist origins of the dramatic expressionism : Gérard de Nerval}

The aim of the article is to show that some motifs and concepts which can be found in the expressionist dramas - emerged in the early part of the $X^{\text {th }}$ century - have already been explored by Gérard de Nerval (1808$1855)$ during the last years of his life. The paper analyses in particular the last - and unfinished - Nerval's récit entitled Aurélia in which analogies with some techniques used by expressionist authors such as the exploration of the universe of dreams and soul or the research of the true identity of man are more than surprising.

\section{keywords}

pre-symbolism, Gérard de Nerval, expressionism, dream, occultism

\section{mots-clés}

pré-symbolisme, Gérard de Nerval, expressionnisme, rêve, occultisme

\section{sebastian ziólkowski}

Étudiant en Master à I'Institut de Philologie Romane de I'Université de Gdańsk. En 2017, il a soutenu le mémoire de licence portant le titre : Le tragique de l'individu dans l'œuvre romanesque de Louis-Ferdinand Céline. Actuellement, il prépare son mémoire de maîtrise intitulé Du présymbolisme à l'expressionnisme dramatique en France. II s'intéresse avant tout à la littérature du XIXe et de la première moitié du XXe siècle et, d'une manière toute particulière, aux avant-gardes françaises du siècle écoulé.

ORCID : http://orcid.org/0000-0001-8763-4951 